\title{
On the Properties of Intermediate Quantifiers and the Quantifier "MORE-THAN"
}

\author{
Vilém Novák ${ }^{(凶)}$, Petra Murinová, and Stefania Boffa \\ Institute for Research and Applications of Fuzzy Modeling, University of Ostrava, \\ NSC IT4Innovations, 30. dubna 22, 70103 Ostrava 1, Czech Republic \\ $\{$ Vilem.Novak, Petra.Murinova, Stefania.Boffa\}@osu.cz
}

\begin{abstract}
This paper continues the research in formal theory of intermediate quantifiers. We present some new properties, introduce intermediate quantifiers of type $\langle 1\rangle$, and also new quantifiers MORE-THAN and LESS-THAN.
\end{abstract}

Keywords: Generalized quantifiers - Fuzzy quantifiers · Fuzzy type theory $\cdot$ Mathematical fuzzy logic

\section{Introduction}

Quantifiers are special expressions of natural language that characterize quantity of objects having a given property. Typical examples are the classical ones "all, exists", but also "most, almost all, many, few", etc. As they are quite frequently used in common language, they raised interest of logicians who tried to suggest logical models of them. The general theory was initiated by Mostowski [8] and further elaborated by many logicians (cf. [21] and citations therein).

A general and widely accepted definition originated by Lindström in [7] is to take quantifiers as $n$-ary relations among subsets of powers of a given set $M$, i.e., subsets of $P\left(M^{k_{1}}\right) \times \cdots \times P\left(M^{k_{n}}\right)$. Adoption of this definition to fuzzy logic is the following (cf. [12]): a generalized fuzzy quantifier of type $\left\langle k_{1}, \ldots, k_{n}\right\rangle$ is a functional $\mathbf{Q}$ that assigns to each non-empty set $M$ a fuzzy relation

$$
Q_{U}: \mathcal{F}\left(M^{k_{1}}\right) \times \cdots \times \mathcal{F}\left(M^{k_{n}}\right) \longrightarrow E
$$

where by $\mathcal{F}(\cdot)$ we denote a set of all fuzzy sets on a given universe and $E$ is a support of the algebra of truth values. Note that (1) is an $n$-ary fuzzy relation over $k_{i}$-ary fuzzy relations, $i=1, \ldots, n$. This definition is semantic which means that (1) interprets a certain formula $Q\left(A_{1}^{k_{1}}, \ldots, A_{n}^{k_{n}}\right)$ of a suitable formal logic where $A_{1}, \ldots, A_{n}$ are formulas and the exponents $A_{i}^{k_{i}}, i=1, \ldots, n$ denote $k_{i}$-ary conjunctions of them.

The work was supported from ERDF/ESF by the project "Centre for the development of Artificial Intelligence Methods for the Automotive Industry of the region" No. CZ.02.1.01/0.0/0.0/17-049/0008414 and partially also by the MŠMT project NPU II project LQ1602 "IT4Innovations excellence in science".

(C) Springer Nature Switzerland AG 2020

M.-J. Lesot et al. (Eds.): IPMU 2020, CCIS 1239, pp. 159-172, 2020.

https://doi.org/10.1007/978-3-030-50153-2_12 
An important class of quantifiers are intermediate ones, for example most, few, almost all, a lot of, many, a great deal of, a large part of, a small part of, etc. Intermediate quantifiers occur in sentences of natural language of the form

$Q B$ s are $A$

where $Q$ is a quantifier and $B, A$ are properties of elements. Example of (2) is the sentence "Most $(Q)$ young people $(B)$ are happy $(A)$ ". Semantics of intermediate quantifiers lays between the two limit cases: the classical general (universal) $\forall$ and the existential $\exists$ ones (hence the name). From the point of view of (1), intermediate quantifiers are special generalized quantifiers of type $\langle 1,1\rangle$ (cf. [6,19]).

An in-depth linguistic and logical analysis of intermediate quantifiers was provided by Peterson in [20]. He specified their basic semantic properties, and, using informal tools, demonstrated that 105 generalized syllogisms with five selected intermediate quantifiers should be valid. These results inspired Novák to develop a mathematical model of the meaning of intermediate quantifiers (see [15]). The primary formal tool is higher-order fuzzy logic (namely, Łukasiewicz fuzzy type theory (FTT)). This logic is a generalization of classical higher-order logic (also called $\lambda$-calculus), and it was chosen because of its very high explication power. Note that the classical $\lambda$-calculus became a standard tool used by linguists when studying the semantic properties of natural language.

The core idea of the mentioned formalization consists in the assumption that intermediate quantifiers can be taken as the classical $\forall$ or $\exists$ quantifiers applied over a universe whose size is characterized by a measure that can be modified and linguistically evaluated. Note that the idea of using the measure in fuzzy quantifiers also occurs in $[3,5])$.

Using formal language, sentence (2) can be construed by a certain formula $(Q x)(B, A)$ where $B$ and $A$ are subformulas representing properties. This formula is precisely defined in Sect. 4 . The theory of intermediate quantifiers is already quite well developed and presented in many papers (see, e.g., [9-11]).

The main objective of this paper is to continue development of the theory of intermediate quantifiers. We introduce simpler quantifiers of type $\langle 1\rangle$ that are time to time needed in some reasoning, and also introduce new intermediate quantifiers MORE-THAN and LESS-THAN, and prove validity of the related generalized syllogisms.

By a fuzzy set in a universe $M_{\alpha}$ we mean a function $A: M_{\alpha} \longrightarrow E$ where $E$ is the support of a suitable algebra of truth values. If $A$ is a fuzzy set on $M_{\epsilon}$ then we write $A \subsetneq M_{\alpha}$. The kernel of $A$ is the set $\operatorname{Ker}(A)=\left\{x \mid x \in M_{\alpha}, A(x)=1\right\}$.

\section{Preliminaries}

\subsection{Fuzzy Type Theory}

The theory of intermediate quantifiers has been developed in Łukasiewicz fuzzy type theory (E-FTT) whose algebra of truth values is a linearly ordered MValgebra. Note that E-FTT is a gneralization of the classical type theory (see [1] and elsewhere). 
The basic syntactical objects of E-FTT are classical, namely the concepts of type and formula. Recall that by type we understand a certain symbol expressing a kind of objects that are denoted by a formula in concern. The types are recursively formed starting with the atomic types $\epsilon$ (elements), and $o$ (truth values). Complex types are defined as follows: if $\alpha, \beta$ are types then $(\beta \alpha)$ is a type. We denote types by Greek letters and the set of all types by Types. Each formula is assigned a type and we write $A_{\alpha}$ where $A$ is a formula and $\alpha$ a type.

The language $J$ of E-FTT consists of variables $x_{\alpha}, \ldots$, special constants $c_{\alpha}, \ldots$ ( $\alpha \in$ Types), the symbol $\lambda$, and brackets. We will consider the following concrete special constants: $\mathbf{E}_{(o \alpha) \alpha}$ (fuzzy equality) for every $\alpha \in$ Types, $\mathbf{C}_{(o o) o}$ (conjunction), $\mathbf{D}_{(o o)}$ (delta operation on truth values) and the description operator $\iota_{\epsilon(o \epsilon)}$.

Formulas are formed of variables, constants (each of specific type), and the symbol $\lambda$. A set of all formulas of type $\alpha$ is denoted by Form $\alpha$. The set of all formulas is Form $=\bigcup_{\alpha \in \text { Types }}$ Form $_{\alpha}{ }^{1}$.

If $B \in$ Form $_{\beta \alpha}$ and $A \in$ Form $_{\alpha}$ then $(B A) \in$ Form $_{\beta}$. Similarly, if $A \in$ Form $_{\beta}$ and $x_{\alpha} \in J, \alpha \in$ Types, is a variable then $\left(\lambda x_{\alpha} A\right) \in$ Form $_{\beta \alpha}$.

A formal theory $T \subset$ Form $_{o}$ is a set of formulas of type $o$ (truth values). Provability is defined classically. If $A_{o}$ is provable in $T$ then we write $T \vdash A_{o}$.

The algebra of truth values of E-FTT is supposed to be a linearly ordered $\mathrm{MV}$-algebra $\langle E, \vee, \wedge, \otimes, \rightarrow, \mathbf{0}, \mathbf{1}, \Delta\rangle$ extended by the operation $\Delta$ (see $[2,18])$. A special case of it is the standard Łukasiewicz $\mathrm{MV}_{\Delta}$-algebra

$$
\mathcal{L}_{\Delta}=\langle[0,1], \vee, \wedge, \otimes, \rightarrow, 0,1, \Delta\rangle
$$

where

$$
\begin{aligned}
\wedge & =\text { minimum, } & \vee & =\text { maximum, } \\
a \otimes b & =0 \vee(a+b-1), & a \rightarrow b & =1 \wedge(1-a+b), \\
\neg a & =a \rightarrow 0=1-a, & \Delta(a) & = \begin{cases}1 & \text { if } a=1, \\
0 & \text { otherwise. }\end{cases}
\end{aligned}
$$

Note that the $\Delta$ operation sends all truth values smaller than 1 to 0 .

A model is $\mathcal{M}=\left\{\left(M_{\alpha}, \stackrel{\circ}{=}_{\alpha}\right) \mid \alpha \in\right.$ Types $\}$ where $\stackrel{\circ}{=}_{\alpha}$ is a fuzzy equality on a set $M_{\alpha}$ (a binary fuzzy relation on $M_{\alpha}$ that is reflexive, symmetric, and $\otimes$-transitive). If $\mathcal{M}$ is a model then $\mathcal{M}\left(A_{o}\right) \in M_{o}$ is a truth value, $\mathcal{M}\left(A_{\epsilon}\right) \in M_{\epsilon}$ is some element and $\mathcal{M}\left(A_{\beta \alpha}\right): M_{\alpha} \longrightarrow M_{\beta}$ is a function. For example, $\mathcal{M}\left(A_{o \alpha}\right): M_{\alpha} \longrightarrow M_{o}$ is a fuzzy set and $\mathcal{M}\left(A_{(o \alpha) \alpha}\right): M_{\alpha} \times M_{\alpha} \longrightarrow M_{o}$ a fuzzy relation. A formula $A_{o}$ is true in $T, T \models A_{o}$, if it is true in the degree 1 in all models of $T$.

The fuzzy type theory is complete, i.e., the completeness theorem stating that a theory $T$ is consistent iff it has a (Henkin) model holds true. We sometimes apply its equivalent version: $T \vdash A_{o}$ iff $T \models A_{o}$.

\footnotetext{
${ }^{1}$ To improve readability of formulas, we quite often write the type only once in the beginning of the formula and then omit it. Alternatively, we write $A \in$ Form $_{\alpha}$ to emphasize that $A$ is a formula of type $\alpha$ and do not repeat its type again.
} 
In the explanation below, we need to characterize that a given formula $A_{o}$ represents a nonzero truth value, and also a general truth value that is neither equal to 0 nor to 1 . The following two formulas will do the job:

$$
\begin{array}{ll}
\Upsilon_{o o} \equiv \lambda z_{o} \cdot \neg \Delta\left(\neg z_{o}\right), & \left(z_{o} \text { is a nonzero truth value }\right) \\
\hat{\Upsilon}_{o o} \equiv \lambda z_{o} \cdot \neg \Delta\left(z_{o} \vee \neg z_{o}\right) & \left(z_{o} \text { is a general truth value }\right) .
\end{array}
$$

It can be proved that $T \models \Upsilon A_{o}$ implies that $\mathcal{M}\left(A_{o}\right)>0$, and $T \models \hat{\Upsilon} A_{o}$ implies that $\mathcal{M}\left(A_{o}\right) \in(0,1)$, in any model $\mathcal{M}$ of the theory $T$.

The following lemma characterizes a few basic properties of $\boldsymbol{\Delta}$ and $\Upsilon$.

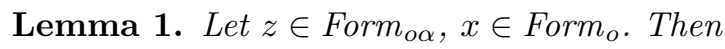

(a) $\vdash \Upsilon(\Delta x) \equiv \Delta x$

(b) $\vdash\left(\left(A_{o} \Rightarrow B_{o}\right) \wedge\left(A_{o} \Rightarrow C_{o}\right)\right) \Rightarrow\left(A_{o} \Rightarrow B_{o} \wedge C_{o}\right)$

(c) $\vdash\left(\left(A_{o} \Rightarrow B_{o}\right) \vee\left(A_{o} \Rightarrow C_{o}\right)\right) \Rightarrow\left(A_{o} \Rightarrow B_{o} \vee C_{o}\right)$

Proof. (a) is obtained by the following sequence of inferences:
$(\mathrm{L} .1) \vdash \Upsilon(\Delta x) \equiv \neg \Delta \neg \Delta x$
(L.2) $\vdash \Delta \perp \equiv \perp$
(L.3) $\vdash \Delta \top \equiv \top$
(L.4) $\vdash \neg \perp \equiv \top$
$(\mathrm{L} .5) \vdash \neg \top \equiv \perp$
$(\mathrm{L} .6) \vdash \Upsilon(\perp) \equiv \perp$
$(\mathrm{L} .7) \vdash \Upsilon(\top) \equiv \top$
$(\mathrm{L} .8) \vdash \Upsilon(\Delta x) \equiv \Delta x$

(definition of $\Upsilon$ )

(properties of $\boldsymbol{\Delta}$ )

(properties of $\boldsymbol{\Delta}$ )

(properties of FTT)

(properties of FTT)

(L.1-L.5 using Rule (R))

(L.1-L.5 using Rule (R))

(L.6, L.7 by [13, Theorem 14])

(b), (c) are proved using the standard means of FTT (cf. also [4, Lemma $2.2 .9])$.

We will also work with the derived connective

$$
\ominus_{(o o) o} \equiv \lambda x_{o} \lambda y_{o} \cdot x \& \neg y
$$

that is in the standard Łukasiewicz algebra interpreted by the operation $a \ominus b=$ $a \otimes \neg b=\max \{0, a-b\}$. Finally, we define the formula

$$
x_{o}<_{(o o) o} y_{o} \equiv\left(x_{o} \Rightarrow y_{o}\right) \& \neg \Delta\left(x_{o} \equiv y_{o}\right) \text {. }
$$

Note that a fuzzy set in a universe $M_{\alpha}$ is in FTT represented by a formula $X_{o \alpha}$. Indeed, let $\mathcal{M}$ be a model. Then $\mathcal{M}\left(\mathcal{A}_{\alpha}\right): M_{\alpha} \longrightarrow E^{2}$. In the same way, a $\lambda$-formula ${ }^{3} \lambda x_{\alpha} B_{o}$ also represents a fuzzy set.

Our explanation below proceeds mostly on the level of syntax because it is the most general way how to express various kinds of properties, and the results are universally valid in all models. W.r.t. the previous paragraph, we will freely call formulas of type $o \alpha$ "fuzzy sets" instead of more precise "formulas (variables) representing fuzzy sets". For example, we say "a fuzzy set $x_{o \alpha}$ " or "a fuzzy set $A_{o \alpha}$ ". The reader, however, should be aware that fuzzy sets are obtained only in a model after proper interpretation of these formulas.

\footnotetext{
${ }^{2}$ Recall that we identify a fuzzy set with its membership function.

${ }^{3}$ In type theory, $\lambda$-formulas are often called $\lambda$-terms.
} 


\subsection{Evaluative Linguistic Expressions}

The theory of intermediate quantifiers is based on the theory of evaluative linguistic expressions that are expressions of natural language such as "small, medium, big, very short, more or less deep, quite roughly strong, extremely high", etc. Semantics of them is also formalized using the language of L-FTT (see [14]). Less formally, including formulas for the direct computation is their theory presented in [17].

The theory of evaluative linguistic expressions is a special formal theory $T^{\mathrm{Ev}}$ of Ł-FTT. Its language $J^{\mathrm{Ev}}$ has the following special symbols:

(i) The constants $\top, \perp \in$ Form $_{o}$ for truth and falsity and $\dagger \in$ Form $_{o}$ for the middle truth value ${ }^{4}$.

(ii) A special constant $\sim \in$ Form $_{(o o) o}$ for an additional fuzzy equality on the set of truth values $E$.

(iii) A set of special constants $\boldsymbol{\nu}, \ldots \in$ Form $_{\text {oo }}$ for linguistic hedges and a set of triples of additional constants $\mathbf{a}_{\boldsymbol{\nu}}, \mathbf{b}_{\boldsymbol{\nu}}, \mathbf{c}_{\boldsymbol{\nu}}, \ldots \in$ Form $_{o}$ where each triple is associated with one hedge $\boldsymbol{\nu}$. The $J^{\mathrm{Ev}}$ is supposed to contain the special constants $\{E x, S i, V e, M L, R o, Q R, V R\}$ that represent the linguistic hedges (extremely, significantly, very, roughly, more or less, rather, quite roughly, very roughly, respectively).

The logical theory of evaluative expressions contains models of the standard logical and linguistic concepts of intension and extension (see [14] for the technical details). Evaluative expressions considered in this paper are construed by special formulas of type oo(oo): Sm (small), Me (medium), Bi (big), and $Z e$ (zero) that can be extended by the linguistic hedges introduced above. For example, $S m V e$ is a formula whose interpretation is intension of the linguistic expression "very small'. If the concrete expression is not important, we use in the sequel a metavariable $E v$ for intension of an arbitrary evaluative expression.

\section{Cuts of Fuzzy Sets}

To define intermediate quantifiers, we need a special operation called cut of a fuzzy set. It is motivated by the need to form a new fuzzy set from a given one by extracting several elements together with their membership degrees and putting the other membership degrees equal to 0. For example, given a fuzzy set $A=\left\{0.3 / x_{1}, 1 / x_{2}, 0.7 / x_{3}, 0.9 / x_{4}\right\}$, we may need to work with its part only, say a fuzzy set $A^{\prime}=\left\{0.3 / x_{1}, 0.9 / x_{4}\right\}$. We thus cut from $A$ the singletons $0.3 / x_{1}$ and $0.9 / x_{4}$ and put them into $A^{\prime}$. The elements of $A^{\prime}$ can be specified by means of some other fuzzy set, say $B=\left\{0.3 / x_{1}, 0.7 / x_{2}, 0.9 / x_{4}\right\}$ whose elements of interest (i.e., $x_{1}, x_{4}$ ) have membership degrees equal to those of $A$. The resulting fuzzy set $A^{\prime}$ is thus obtained by a cut of $A$ by $B$, i.e., $A^{\prime}=A \mid B$.

\footnotetext{
$\overline{4}$ The formula $\dagger$ is in the standard Eukasiewicz MV-algebra interpreted by the value 0.5 .
} 
This operation is formally defined as follows: Let $y, z \in$ Form $_{o \alpha}$ be variables of type $o \alpha, \alpha \in$ Types. The cut of $y_{o \alpha}$ by $z_{o \alpha}$ is the fuzzy set

$$
y_{o \alpha} \mid z_{o \alpha} \equiv \lambda x_{\alpha} \cdot z_{o \alpha} x_{\alpha} \& \Delta\left(\Upsilon\left(z_{o \alpha} x_{\alpha}\right) \Rightarrow\left(y_{o \alpha} x_{\alpha} \equiv z_{o \alpha} x_{\alpha}\right)\right) .
$$

This formula says the following: the fuzzy set $y \mid z$ is a function that to each $x_{\alpha}$ assigns a truth value of the conjunction of truth values $z x^{5}$ and $\Delta(\Upsilon(z x) \Rightarrow$ $(y x \equiv z x))$ where the latter has the truth value 1 , if $z x$ is nonzero and the truth values $y x$ and $z x$ are equal. Otherwise, it has the truth value 0 .

The following lemma shows that thus defined operation does precisely what we want.

Lemma 2 ([11]). Let $\mathcal{M}$ be a model and $p$ an assignment of elements to variables $y_{o \alpha}, z_{o \alpha}$ such that $\mathcal{M}_{p}(y)=B \Subset M_{\alpha}, \mathcal{M}_{p}(z)=Z \subsetneq M_{\alpha}$. Then for any $m \in M_{\alpha}$

$$
\mathcal{M}_{p}(y \mid z)(m)=(B \mid Z)(m)= \begin{cases}B(m), & \text { if } B(m)=Z(m), \\ 0 & \text { otherwise. }\end{cases}
$$

Let us also introduce the following special fuzzy sets: A fuzzy set $X_{o \alpha}$ is crisp if it has the property

$$
\operatorname{Crisp}_{o(o \alpha)} X_{o \alpha} \equiv\left(\forall u_{\alpha}\right)(X u \equiv \Delta(X u))
$$

Hence, a crisp fuzzy set has elements with membership degrees equal either to 1 or 0 .

Support of a fuzzy set $X_{o \alpha}$ is a set defined by

$$
\operatorname{Supp}_{(o \alpha)(o \alpha)} X_{o \alpha} \equiv \lambda u_{\alpha} \cdot \Upsilon\left(X_{o \alpha} u_{\alpha}\right)
$$

The universal set is defined by

$$
V_{\alpha} \equiv \lambda x_{\alpha} \top
$$

Note that interpretation of $V_{\alpha}$ in a model $\mathcal{M}$ is $\mathcal{M}\left(V_{\alpha}\right)=M_{\alpha}$.

The following are basic properties of cut.

Lemma 3. Let $B \in$ Form $_{o \alpha}, \alpha \in$ Types.

(a) $\vdash B \mid B \equiv B$.

(b) $\vdash B \mid \emptyset \equiv \emptyset$.

(c) $\vdash \operatorname{Crisp} \emptyset_{o \alpha}$.

(d) $\vdash \operatorname{Crisp}(\operatorname{Supp} B)$.

(e) $\operatorname{Crisp} B \vdash(V \mid B \equiv B)$.

(f) $\operatorname{Crisp} B \vdash\left(\forall z_{o \alpha}\right) \operatorname{Crisp}(B \mid z)$.

(g) $B \subset B^{\prime} \vdash(\exists z) \neg\left(B\left|z \subseteq B^{\prime}\right| z\right)$.

${ }^{5}$ In fact, $z_{o \alpha} x_{\alpha}$ is a membership degree of $x_{\alpha}$ in $z_{o \alpha}$. 
Proof. (a), (b) are proved in [11], (c), (d) in [16].

(e)

$$
\begin{array}{lr}
(\text { L.1 }) \vdash(V \mid B) x \equiv B x \& \Delta(\Upsilon(B x) \Rightarrow(V x \equiv B x)) & \text { (definition of } \mid) \\
\text { (L.2) } \vdash B x \equiv \Delta B x & \text { (assumption) } \\
\text { (L.3) } \vdash(V \mid B) x \equiv B x \& \Delta(\Upsilon(\Delta(B x)) \Rightarrow(\top \equiv \Delta(B x))) & \text { (L.1, L.2, Rule (R)) } \\
\text { (L.4) } \vdash(V \mid B) x \equiv B x \& \Delta(\Delta(B x) \Rightarrow(\Delta(B x))) & \text { (L.3, Lemma 1(a), Rule (R)) } \\
\text { (L.5) } \vdash(V \mid B) x \equiv B x & \text { (L.4, properties of FTT) }
\end{array}
$$

(g) Let $\mathbf{v}$ be a constant, and $z_{o \alpha}$ a fuzzy set such that $\vdash\left(B \mathbf{v}<B^{\prime} \mathbf{v}\right) \wedge(z \mathbf{v} \equiv$ $B \mathbf{v})$. Then $\vdash\left(B^{\prime} \mid z\right) \mathbf{v} \equiv \perp$ which implies (g) using the $\exists$-substitution axiom.

\section{Intermediate Quantifiers}

The theory of intermediate quantifiers is based on the concepts of measure of a fuzzy set, and linguistic evaluation of its size. All technical details not mentioned in this paper can be found in [11].

The measure is defined below. Note that we consider a relative measure, i.e., a measure of a fuzzy set $x_{o \alpha}$ w.r.t. a fuzzy set $z_{o \alpha}$.

Definition 1. Let $R \in$ Form $_{o(o \alpha)(o \alpha)}$ be a formula ${ }^{6}$.

(i) A formula $\mu \in$ Form $_{o(o \alpha)(o \alpha)}$ defined by

$$
\mu_{o(o \alpha)(o \alpha)}:=\lambda z_{o \alpha} \lambda x_{o \alpha}\left(R z_{o \alpha}\right) x_{o \alpha}
$$

represents a measure on fuzzy sets in the universe of type $\alpha \in$ Types if it has the following properties:

$$
\begin{aligned}
& \text { (M1) } \Delta\left(x_{o \alpha} \subseteq z_{o \alpha}\right) \& \Delta\left(y_{o \alpha} \subseteq z_{o \alpha}\right) \& \Delta\left(x_{o \alpha} \subseteq y_{o \alpha}\right) \Rightarrow \\
& \left(\left(\mu z_{o \alpha}\right) x_{o \alpha} \Rightarrow\left(\mu z_{o \alpha}\right) y_{o \alpha}\right), \\
& \text { (M2) } \Delta\left(x_{o \alpha} \subseteq z_{o \alpha}\right) \Rightarrow\left(\left(\mu z_{o \alpha}\right)\left(z_{o \alpha} \backslash x_{o \alpha}\right) \equiv \neg\left(\mu z_{o \alpha}\right) x_{o \alpha}\right), \\
& \text { (M3) } \Delta\left(x_{o \alpha} \subseteq y_{o \alpha}\right) \& \Delta\left(x_{o \alpha} \subseteq z_{o \alpha}\right) \& \Delta\left(y_{o \alpha} \subseteq z_{o \alpha}\right) \Rightarrow \\
& \quad\left(\left(\mu z_{o \alpha}\right) x_{o \alpha} \Rightarrow\left(\mu y_{o \alpha}\right) x_{o \alpha}\right) .
\end{aligned}
$$

(ii) The following formula characterizes measurable fuzzy sets of a given type $\alpha$ :

$$
\begin{aligned}
\mathbf{M}_{o(o \alpha)}:=\lambda z_{o \alpha} \cdot \neg \Delta\left(z_{o \alpha} \equiv \emptyset_{o \alpha}\right) \& \Delta\left(\mu z_{o \alpha}\right) z_{o \alpha} \& \\
\left(\forall x_{o \alpha}\right)\left(\forall y_{o \alpha}\right) \Delta((M 1) \&(M 3)) \&\left(\forall x_{o \alpha}\right) \Delta(M 2)
\end{aligned}
$$

where, for the simplicity of expression, we write (M1)-(M3) to stand for the axioms from (i).

\footnotetext{
${ }^{6}$ This formula can be understood as a procedure for computation of the output on the basis of a given input. In our case, the output is size (element of $E$ ) of the measure of a fuzzy set with respect to another one.
} 
Axioms (M1) and (M3) characterize monotonicity of measure; namely that it is isotone w.r.t. $x_{o \alpha}$ and antitone w.r.t. $z_{o \alpha}$. Axiom (M2) characterizes measure of a complement of $x_{o \alpha}$ w.r.t. $z_{o \alpha}$.

We consider a formal theory $T^{\mathrm{IQ}}$ in which intermediate quantifiers are definable in the sense of the definition below. The theory must contain the theory of evaluative expressions and measurable fuzzy sets (see [11] for the details).

Definition 2. Let $T^{I Q}$ be a theory containing intermediate quantifiers and $E v \in$ Form $_{\text {oo }}$ be an intension of some evaluative linguistic expression. Finally, let $z \in$ Form $_{o \alpha}, x \in$ Form $_{\alpha}$ be variables, and $A, B \in$ Form $_{o \alpha}$ be formulas where $T^{I Q} \vdash \mathbf{M}_{o(o \alpha)} B_{o \alpha}$. An intermediate generalized quantifier is one of the following formulas:

$$
\begin{aligned}
& \left(Q_{E v}^{\forall} x_{\alpha}\right)(B, A) \equiv(\exists z)[(\forall x)((B \mid z) x \Rightarrow A x) \wedge E v((\mu B)(B \mid z))], \\
& \left(Q_{E v}^{\exists} x_{\alpha}\right)(B, A) \equiv(\exists z)[(\exists x)((B \mid z) x \wedge A x) \wedge E v((\mu B)(B \mid z))] .
\end{aligned}
$$

Either of the quantifiers (7) or (8) construes the sentence

\section{"〈Quantifier $\rangle$ 's are A"}

where $\left\langle\right.$ Quantifier〉 is some intermediate quantifier. The formula $B_{o \alpha}$ represents a universe of quantification and $A_{o \alpha}$ a property of elements of type $\alpha$.

Formula (7) has a clear meaning: all elements from a certain part $z_{o \alpha}$ of the universe $B_{o \alpha}$ have the property $A_{o \alpha}$, and size of the cut $B \mid z$ w.r.t. whole $B$ is linguistically evaluated by $E v$. For example the sentence "Most $B$ are $A$ " is construed by the formula $\left(Q_{B i V e}^{\forall} x\right)(B, A)$ which, using (7), is equivalent to $(\exists z)[(\forall x)((B \mid z) x \Rightarrow A x) \wedge(B i V e)((\mu B)(B \mid z))]$. Similarly for $(8)$.

Remark 1. The original definition of intermediate quantifiers introduced, e.g., in [15] considered all fuzzy subsets $z_{o \alpha}$ of $B_{o \alpha}$. When computing intermediate quantifiers on real data, however, it turned out that we obtain counterintuitive results. For example, "Most young women have long hair". If a given woman is young in the degree 0.7 , it would be strange to consider her to be young in the degree 0.3 . This is the reason why we introduced the operation of cut in $(7)$ and (8).

Theorem 1. Let $A, B, C, z \in$ Form $_{o \alpha}$ be formulas representing properties of objects and $E v \in$ Form $_{o o}$ be an intension of some evaluative expression.

(a) $T^{I Q} \cup\{A \subseteq C\} \vdash\left(Q_{E v}^{\forall} x_{\alpha}\right)(B, A) \Rightarrow\left(Q_{E v}^{\forall} x_{\alpha}\right)(B, C)$,

(b) $T^{I Q} \vdash\left(Q_{E v}^{\forall} x_{\alpha}\right)(B, A) \vee\left(Q_{E v}^{\forall} x_{\alpha}\right)(B, C) \Rightarrow\left(Q_{E v}^{\forall} x_{\alpha}\right)(B, A \cup C)$.

Proof. (a) follows from and $\vdash\left(\forall x_{\alpha}\right)(B x \Rightarrow A x) \Rightarrow\left(\forall x_{\alpha}\right)(B x \Rightarrow C x)$ and the assumption. 
(b) Using Lemma 1(b) and the properties of FTT, we can prove that

$$
\vdash\left(\forall x_{\alpha}\right)((B \mid z) x \Rightarrow A x) \vee\left(\forall x_{\alpha}\right)((B \mid z) x \Rightarrow c x) \Rightarrow\left(\forall x_{\alpha}\right)((B \mid z) x \Rightarrow A x \wedge C x)
$$

Adding $\operatorname{Ev}((\mu B)(B \mid z))$ to both sides of this implication, we obtain valid implication. Then, using distributivity of $\vee, \wedge$ and the property $\vdash(\exists z)(P z \vee Q z) \Rightarrow$ $((\exists z) P z \vee(\exists z) Q z)$ we obtain (b).

The quantifiers defined above are of type $\langle 1,1\rangle$. It is possible, however, to introduce also simpler quantifiers of type $\langle 1\rangle$ that make quantification over the whole universe (similarly as the classical quantifiers $\forall$ and $\exists$ do).

Definition 3 (Quantifiers of type $\langle 1\rangle$ ). Let $A \in$ Form $_{o \alpha}, x \in$ Form $_{\alpha}$ and $V_{o \alpha}$ be the universal set (4). Let $\vdash \mathbf{M}\left(V_{o \alpha}\right)$. Then the formula

$$
\left(Q_{E v}^{\forall} x\right) A \equiv\left(Q_{E v}^{\forall} x\right)(V, A) \equiv(\exists z)[(\forall x)((V \mid z) x \Rightarrow A x) \wedge E v((\mu V)(V \mid z))]
$$

is an intermediate quantifier of type $\langle 1\rangle^{7}$.

Theorem 2. Let $A, z \in$ Form $_{o \alpha}$ and $E v \in\{B i \boldsymbol{\nu} \mid \boldsymbol{\nu} \in\{E x, S i, V e, M L, R o, Q R$, $V R\}\}$. Then

$$
\left(Q_{E v}^{\forall} x\right) A \equiv(\exists z)[(\forall x)((\operatorname{Supp} A \mid z) x \Rightarrow A x) \wedge E v((\mu V)(\operatorname{Supp} A \mid z)) .
$$

Proof. By Lemma 3(f), $V \mid z$ is crisp for all fuzzy sets $z \in$ Form $_{o \alpha}$. Let $\mathcal{M}$ be a model, $p$ an assignment, and $z_{o \alpha}$ be such that $\mathcal{M}_{p}(V \mid z) \cap\left(M_{\alpha} \backslash \mathcal{M}_{p}(\operatorname{Supp} A)\right) \neq \emptyset$. Then $\mathcal{M}_{p}((\forall x)((V \mid z) x \Rightarrow A x))=0$. From it follows that the latter is (in general) non-zero only if $\mathcal{M}_{p}(V \mid z) \subseteq \mathcal{M}_{p}(\operatorname{Supp} A)$.

This theorem suggests a simplified way how intermediate quantifiers of type $\langle 1\rangle$ can be computed. Namely, it is sufficient to confine only to the support of parts of $A$.

\section{The Quantifier "MORE-THAN"}

This kind of quantifier is studied in the theory of generalized quantifiers $[6,19]$. An example of such quantifier is the following:

$$
\text { More girls than boys are diligent. }
$$

Classical model of this quantifier is

$$
M T(B, C, A)=1 \quad \text { iff } \quad|B \cap A|>|C \cap A|
$$

where $M T$ is the quantifier and $|\cdot|$ denotes number of elements (in a finite set). This definition does not consider hedging, i.e., modifying by hedges such as

\footnotetext{
${ }^{7}$ From the point of view of general theory, this quantifier trivially fulfills the property of relativization.
} 
"much" or "a lot of". Introducing them would already require specification of the context w.r.t. which we could specify, how much greater $|B \cap A|$ than $|C \cap A|$ should be. This problem is solved in our definition below, in which we explicitly consider a universe $U$.

Note that in (10), we do not claim that all girls are more diligent than all boys; only certain part of girls are diligent and the same for boys. We compare sizes of these (fuzzy) sets w.r.t. a certain universe $U$ that can be, e.g., all children at school, or a some more specific part of them.

Definition 4. Let $A, B, C, z_{1}, z_{2} \in$ Form $_{o \alpha}$ be formulas representing properties of objects and $U \in$ Form $_{o \alpha}$ be a measurable universe, i.e., $T^{I Q} \vdash \mathbf{M}(U)$ holds. Let $T^{I Q} \vdash(B \subseteq U) \wedge(C \subseteq U)$. Then:

(i) The expression

More $B$ than $C$ are $A$ (in a universe $U$ )

is construed by the formula

$$
\begin{gathered}
\left(M T^{\forall} x_{\alpha}\right)(B, C, A ; U) \equiv\left(\exists z_{1}\right)\left(\exists z_{2}\right)\left[\left(\forall x_{\alpha}\right)\left(\left(B \mid z_{1}\right) x \Rightarrow A x\right) \wedge\right. \\
\left.\left(\forall x_{\alpha}\right)\left(\left(C \mid z_{2}\right) x \Rightarrow A x\right) \wedge \Upsilon\left((\mu U)\left(B \mid z_{1}\right) \ominus(\mu U)\left(C \mid z_{2}\right)\right)\right] .
\end{gathered}
$$

(ii) The modified expression of the form

$\langle$ Hedge $\rangle$ more $B$ than $C$ are $A$ (in a universe $U$ )

where $\langle$ Hedge $\rangle$ can be, e.g., "much", "a lot", "a little", etc. In formal language, it is construed by the formula

$$
\begin{aligned}
M T_{E v}^{\forall}(B, C, A ; U) \equiv\left(\exists z_{1}\right)\left(\exists z_{2}\right)\left[(\forall x)\left(\left(B \mid z_{1}\right) x \Rightarrow A x\right) \wedge\right. \\
\left.(\forall x)\left(\left(C \mid z_{2}\right) x \Rightarrow A x\right) \wedge(E v)((\mu U)(B \mid z) \ominus(\mu U)(C \mid z))\right] .
\end{aligned}
$$

The evaluative expression $E v$ can be the following:

(i) $\langle$ Hedge $\rangle:=$ Much: we put $E v:=B i \overline{\boldsymbol{\nu}}$

(ii) $\langle$ Hedge $\rangle:=$ Very much: we put $E v:=B i$ Ve

(iii) $\langle$ Hedge $\rangle:=A$ little: we put $E v:=S m V e$

$$
\begin{aligned}
& \text { (simple "big"), } \\
& \text { ("very big"), } \\
& \text { ("very small"). }
\end{aligned}
$$

Analogously, we can define other similar kinds of hedges in (ii).

There is one problem with this definition. When analyzing the linguistic expression (10), we see that it internally consists of two propositions:

(a) Girls are diligent.

(b) Boys are diligent.

In our definition we suggest to model both expressions by the implication

$$
(\forall x)(B x \Rightarrow A x) \text {. }
$$


The classical definition (11), however, would suggest the model

$$
(\exists x)(B x \wedge A x) .
$$

In this case, Definition (12) would change into

$$
\begin{aligned}
\left(M T^{\exists} x_{\alpha}\right)(B, C, A ; U) & \equiv\left(\exists z_{1}\right)\left(\exists z_{2}\right)\left[(\exists x)\left(\left(B \mid z_{1}\right) x \wedge A x\right) \wedge\right. \\
(\exists x)\left(\left(C \mid z_{2}\right) x \wedge A x\right) & \Upsilon \Upsilon((\mu U)(B \mid z) \ominus(\mu U)(C \mid z))] .
\end{aligned}
$$

We need more investigation to give the definite answer. At this moment, let us only remark that definition (12) corresponds to (7).

Theorem 3. Let $A, B, C \in$ Form $_{o \alpha}$ be formulas. Let $T^{I Q} \vdash\left(Q_{E v}^{\forall} x\right)(C, A)$ where $E v \in\{\neg S m \overline{\boldsymbol{\nu}}, B i$ Ve, Bi Ex $\}$ and $T^{I Q} \vdash\left(M T^{\forall} x_{\alpha}\right)(B, C, A ; U)$. Then $T^{I Q} \vdash$ $\left(Q_{E v}^{\forall} x\right)(B, A)$.

Proof. Semantic: Let $\mathcal{M}$ be a model of $T^{\mathrm{IQ}}$. For better readability, we put $\mathcal{M}_{p}\left(z_{1}\right)=Z_{1}, \mathcal{M}_{p}\left(z_{2}\right)=Z_{2}, \mathcal{M}_{p}(B)=B, \mathcal{M}_{p}(C)=C, \mathcal{M}_{p}(U)=U$, and similarly the other symbols.

By the assumption,

$$
\begin{gathered}
\bigvee_{\mathcal{M}_{p}\left(z_{2}\right)=Z_{2} \bigodot_{M_{\alpha}}} \mathcal{M}_{p}\left((\forall x)\left(\left(C \mid z_{2}\right) x \Rightarrow A x\right)\right) \wedge \operatorname{Ev}\left((\mu C)\left(C \mid Z_{2}\right)\right)=1, \\
\bigvee_{\mathcal{M}_{p}\left(z_{1}\right)=Z_{1} \bigodot_{\sim} M_{\alpha} \mathcal{M}_{p}\left(z_{2}\right)=Z_{2} \bigodot_{\sim} M_{\alpha}}\left[\mathcal{M}_{p}\left((\forall x)\left(\left(B \mid z_{1}\right) x \Rightarrow A x\right)\right) \wedge\right. \\
\left.\mathcal{M}_{p}\left((\forall x)\left(\left(C \mid z_{2}\right) x \Rightarrow A x\right)\right) \wedge \Upsilon\left((\mu U)\left(B \mid Z_{1}\right) \ominus(\mu U)\left(C \mid Z_{2}\right)\right)\right]=1
\end{gathered}
$$

Hence, to every $a, 0<a \leq 1$, we have $a \leq$ (15), and also $a \leq$ (16). The latter means that there are $Z_{1}, Z_{2} \subsetneq M_{\alpha}$ such that

$$
(\mu U)\left(C \mid Z_{2}\right)<(\mu U)\left(B \mid Z_{1}\right) .
$$

Furthermore, $B, C \subseteq U$. Let us consider $a=(\mu U)\left(C \mid Z_{2}\right)$ for some $Z_{2}$. Then $a \leq$ $E v\left((\mu C)\left(C \mid Z_{2}\right)\right)$ because $(\mu U)\left(C \mid Z_{2}\right) \leq(\mu C)\left(C \mid Z_{2}\right)$ by the properties of measure. But then by (17) and the properties of measure, we obtain $a \leq(\mu B)\left(B \mid Z_{1}\right)$ for some $Z_{1}$ which means that $a \leq E v\left((\mu B)\left(B \mid Z_{1}\right)\right)$ because of the increasing character of the assumed $E v$. Since we considered arbitrary $0<a \leq 1$, we conclude that $\bigvee_{\mathcal{M}_{p}\left(z_{1}\right)=Z_{1}} \bigodot_{M_{\alpha}} \mathcal{M}_{p}\left((\forall x)\left(\left(B \mid z_{1}\right) x \Rightarrow A x\right)\right) \wedge \operatorname{Ev}\left((\mu B)\left(B \mid Z_{1}\right)\right)=1$

By this theorem, if we surely know, e.g., that "More $B$ than $C$ are $A$ " and "Most $C$ are $A$ " then it is valid to conclude that surely "Most (Almost all, All) $B$ are $A$ ".

From the previous theorem, validity of the following (weak) ${ }^{8}$ syllogisms immediately follows.

\footnotetext{
${ }^{8}$ By a strong syllogism with a major premise $A_{o}$, a minor premise $B_{o}$, and a conclusion $C_{o}$ we understand provability of the formula $\vdash A_{o} \& B_{o} \Rightarrow C_{o}$. Note that the syllogism in Corollary 1 is weaker.
} 


\section{Corollary 1.}

$$
\begin{aligned}
& T^{I Q} \vdash\left(Q_{E v}^{\forall} x\right)(C, A) \\
& \frac{T^{I Q} \vdash\left(M T^{\forall} x_{\alpha}\right)(B, C, A ; U)}{T^{I Q} \vdash\left(Q_{E v}^{\forall} x\right)(B, A)}
\end{aligned}
$$

where $E v \in\{\neg S m \overline{\boldsymbol{\nu}}, B i$ Ve, Bi Ex $\}$.

We can also symmetrically consider the quantifier

Less $C$ than $B$ are $A$ (in a universe $U$ )

which can be defined by

$$
\left(L T^{\forall} x_{\alpha}\right)(C, B, A ; U) \equiv\left(M T^{\forall} x_{\alpha}\right)(B, C, A ; U) .
$$

Of course, we can also add 〈Hedge to this quantifier.

By similar arguments as in Theorem 3, we can prove the following.

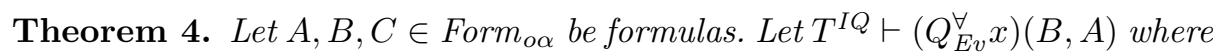
$E v \in\left\{{ }^{+} S m V e,{ }^{+} S m S i\right\}$ and $T^{I Q} \vdash L T_{U}(C, B, A)$. Then $T^{I Q} \vdash\left(Q_{E v}^{\forall} x\right)(C, A)$.

\section{Corollary 2.}

$$
\begin{aligned}
& T^{I Q} \vdash\left(Q_{E v}^{\forall} x\right)(B, A) \\
& \frac{T^{I Q} \vdash\left(L T^{\forall} x_{\alpha}\right)(C, B, A ; U)}{T^{I Q} \vdash\left(Q_{E v}^{\forall} x\right)(C, A)}
\end{aligned}
$$

where $Q \in\{$ A few, Several $\}$.

We may now ask whether the quantifier MORE-THAN is transitive as in the following example:

More young girls than young boys like yoga.

More young boys than managers like yoga.

More young girls than managers like yoga.

Theorem 5. Let $A, B, C, D \in$ Form $_{o \alpha}$ be formulas. Let $T^{I Q} \vdash\left(M T^{\forall} x_{\alpha}\right)(B, C$, $A ; U)$ and $T^{I Q} \vdash\left(M T^{\forall} x_{\alpha}\right)(C, D, A ; U)$. Then $T^{I Q} \vdash\left(M T^{\forall} x_{\alpha}\right)(B, D, A ; U)$.

Proof. Semantic: Let $\mathcal{M}$ be a model of $T^{\mathrm{IQ}}$. For better readability, we put $\mathcal{M}_{p}\left(z_{1}\right)=Z_{1}, \mathcal{M}_{p}\left(z_{2}\right)=Z_{2}, \mathcal{M}_{p}\left(z_{3}\right)=Z_{3}, \mathcal{M}_{p}(A)=A, \mathcal{M}_{p}(D)=D$, $\mathcal{M}_{p}(B)=B, \mathcal{M}_{p}(C)=C, \mathcal{M}_{p}(U)=U$.

By the assumption,

$$
\begin{gathered}
\bigvee_{\mathcal{M}_{p}\left(z_{1}\right)=Z_{1} \subsetneq M_{\alpha} \mathcal{M}_{p}\left(z_{2}\right)=Z_{2} \subsetneq M_{\alpha}}\left[\mathcal{M}_{p}\left((\forall x)\left(\left(B \mid z_{1}\right) x \Rightarrow A x\right)\right) \wedge\right. \\
\left.\mathcal{M}_{p}\left((\forall x)\left(\left(C \mid z_{2}\right) x \Rightarrow A x\right)\right) \wedge \Upsilon\left((\mu U)\left(B \mid Z_{1}\right) \ominus(\mu U)\left(C \mid Z_{2}\right)\right)\right]=1 .
\end{gathered}
$$




$$
\begin{aligned}
& \text { V } \quad\left[\mathcal{M}_{p}\left((\forall x)\left(\left(C \mid z_{2}\right) x \Rightarrow A x\right)\right) \wedge\right. \\
& \mathcal{M}_{p}\left(z_{2}\right)=Z_{2} \subseteq M_{\alpha} \mathcal{M}_{p}\left(z_{3}\right)=Z_{3} \subseteq M_{\alpha} \\
& \left.\mathcal{M}_{p}\left((\forall x)\left(\left(D \mid z_{3}\right) x \Rightarrow A x\right)\right) \wedge \Upsilon\left((\mu U)\left(C \mid Z_{2}\right) \ominus(\mu U)\left(D \mid Z_{3}\right)\right)\right]=1 .
\end{aligned}
$$

Similarly as above, to every $a, 0<a \leq 1$, one can see that $a \leq(18)$ and $a \leq$ (19). From these assumptions it follows that there are $Z_{1}, Z_{2}, Z_{3} \subsetneq M_{\alpha}$ such that

$$
(\mu U)\left(C \mid Z_{2}\right)<(\mu U)\left(B \mid Z_{1}\right) \text { as well as }(\mu U)\left(D \mid Z_{3}\right)<(\mu U)\left(C \mid Z_{2}\right)
$$

which implies that

$$
(\mu U)\left(D \mid Z_{3}\right)<(\mu U)\left(D \mid Z_{1}\right) .
$$

By the properties of E-FTT we have

$$
\begin{array}{r}
\mathcal{M}_{p}\left((\forall x)\left(\left(B \mid z_{1}\right) x \Rightarrow A x\right)\right) \wedge \mathcal{M}_{p}\left((\forall x)\left(\left(C \mid z_{2}\right) x \Rightarrow A x\right)\right) \leq \\
\mathcal{M}_{p}\left((\forall x)\left(\left(B \mid z_{1}\right) x \Rightarrow A x\right)\right) \\
\left.\mathcal{M}_{p}\left((\forall x)\left(\left(C \mid z_{2}\right) x \Rightarrow A x\right)\right) \wedge \mathcal{M}_{p}\left((\forall x)\left(\left(D \mid z_{3}\right) x \Rightarrow A x\right)\right)\right] \leq \\
\mathcal{M}_{p}\left((\forall x)\left(\left(D \mid z_{3}\right) x \Rightarrow A x\right)\right)
\end{array}
$$

Considering arbitrary $0<a \leq 1$ and using (21), (22), (23) we conclude that

$$
\begin{array}{r}
\bigvee_{\mathcal{M}_{p}\left(z_{1}\right)=Z_{1} \subseteq M_{\alpha} \mathcal{M}_{p}\left(z_{3}\right)=Z_{3} \subseteq M_{\alpha}}\left[\mathcal{M}_{p}\left((\forall x)\left(\left(B \mid z_{1}\right) x \Rightarrow A x\right)\right) \wedge\right. \\
\left.\mathcal{M}_{p}\left((\forall x)\left(\left(D \mid z_{3}\right) x \Rightarrow A x\right)\right) \wedge \Upsilon\left((\mu U)\left(B \mid Z_{1}\right) \ominus(\mu U)\left(D \mid Z_{3}\right)\right)\right]=1
\end{array}
$$

by the properties of supremum.

\section{Conclusion}

In this paper, we continued the research in the formal theory of intermediate quantifiers. We proved a few new results, introduced intermediate quantifiers of type $\langle 1\rangle$, and also new quantifiers MORE-THAN and LESS-THAN. We also proved validity of weak syllogisms with these quantifiers.

The future study will be focused on new forms of generalized syllogisms with the proposed quantifiers. Note that they can be used for a linguistic summarization in human reasoning.

\section{References}

1. Andrews, P.: An Introduction to Mathematical Logic and Type Theory: To Truth Through Proof. Kluwer, Dordrecht (2002) 
2. Cignoli, R.L.O., D'Ottaviano, I.M.L., Mundici, D.: Algebraic Foundations of ManyValued Reasoning. Kluwer, Dordrecht (2000)

3. Dvořák, A., Holčapek, M.: L-fuzzy quantifiers of the type $\langle 1\rangle$ determined by measures. Fuzzy Sets Syst. 160, 3425-3452 (2009)

4. Hájek, P.: Metamathematics of Fuzzy Logic. Kluwer, Dordrecht (1998)

5. Holčapek, M.: Monadic L-fuzzy quantifiers of the type $\left\langle 1^{n}, 1\right\rangle$. Fuzzy Sets Syst. 159, 1811-1835 (2008)

6. Keenan, E., Westerståhl, D.: Quantifiers in formal and natural languages. In: van Benthem, J., ter Meulen, A. (eds.) Handbook of Logic and Language, pp. 837-893. Elsevier, Amsterdam (1997)

7. Lindström, P.: First order predicate logic with generalized quantifiers. Theoria 32, 186-195 (1966)

8. Mostowski, A.: On a generalization of quantifiers. Fundamenta Mathematicae 44, 12-36 (1957)

9. Murinová, P., Novák, V.: A formal theory of generalized intermediate syllogisms. Fuzzy Sets Syst. 186, 47-80 (2012)

10. Murinová, P., Novák, V.: The structure of generalized intermediate syllogisms. Fuzzy Sets Syst. 247, 18-37 (2014)

11. Murinová, P., Novák, V.: The theory of intermediate quantifiers in fuzzy natural logic revisited and the model of "Many". Fuzzy Sets Syst. 388, 56-89 (2020)

12. Novák, V.: Antonyms and linguistic quantifiers in fuzzy logic. Fuzzy Sets Syst. 124, 335-351 (2001)

13. Novák, V.: On fuzzy type theory. Fuzzy Sets Syst. 149, 235-273 (2005)

14. Novák, V.: A comprehensive theory of trichotomous evaluative linguistic expressions. Fuzzy Sets Syst. 159(22), 2939-2969 (2008)

15. Novák, V.: A formal theory of intermediate quantifiers. Fuzzy Sets Syst. 159(10), 1229-1246 (2008)

16. Novák, V.: Topology in the alternative set theory and rough sets via fuzzy type theory. Mathematics 8(3), 432-453 (2020)

17. Novák, V., Perfilieva, I., Dvořák, A.: Insight into Fuzzy Modeling. Wiley, Hoboken (2016)

18. Novák, V., Perfilieva, I., Močkoř, J.: Mathematical Principles of Fuzzy Logic. Kluwer, Boston (1999)

19. Peters, S., Westerståhl, D.: Quantifiers in Language and Logic. Clarendon Press, Oxford (2006)

20. Peterson, P.: Intermediate Quantifiers. Logic, linguistics, and Aristotelian semantics. Ashgate, Aldershot (2000)

21. Westerståhl, D.: Quantifiers in formal and natural languages. In: Gabbay, D., Guenthner, F. (eds.) Handbook of Philosophical Logic, vol. IV, pp. 1-131. D. Reidel, Dordrecht (1989) 